Nachstehende reinhistorische Abhandlung wurde 1847 oder Anfangs 1848 von mir, nach Verabredung mit dem verstorbenen Professor Henschel in Breslau, speciell für dessen Zeitschrift „Janus” abgefasst und ihm direct übersandt. Nach seinem kurze Zeit darauf erfolgten allzufrühen Tode wurde mir das Manuscript vom Verleger zurückgeschickt. Seitdem blieb es unverändert und unberührt liegen, da keine andere so speciell medicinischhistorische Zeitschrift existirte. In Cunier's „Annales d'oculistique" hatte ich, schon einige Zeit vor Abfassung dieser ausführlichen Arbeit, den Gegenstand in Kürze abgehandelt, nach der Natur und dem Bedürfnisse dieser, mehr der Praxis als der Geschichte der Augenheilkunde bestimmten Zeitschrift.

Seitdem sind $z$ wanzig Jahre verstrichen, während welcher ich diesen geschichtlichen Punkt der Augenheilkunde und meine Abhandlung vollkommen aus dem Gesichte verlor, bis er vor etwa anderthalb Jahren zufällig zwischen meinem Freunde Prof. A. v. Graefe und mir wieder zur Sprache kam. v. Graefe glaubte, es sei der Mühe werth, diese von mir vergessene Arbeit in einer von dem augenheilkundigen Publikum gelesenen Zeitschrift zu veröffentlichen. Sein Antrag wurde von mir mit Vergnügen angenommen, denn welcher Vater 
lässt gern seine Geisteskinder eines frühen Todes sterben, so unvollkommen und missgestaltet sie auch sein mögen? Und so erscheint denn meine so lange bei Seite gelegte und fast von mir selbst vergessene Abhandlung hier ganz so, wie ich sie vor zwei Jahrzehnten geschrieben. Es wird jedoch nicht übertlüssig sein, ihr einige kurze Bemerkungen vorauszuschicken.

Laugier hat, so viel ich weiss, später nicht inehr von seiner, von ihm als neu betrachteten Operationsmethode gesprochen, welche ich gleich von vornherein als eine. höchst unpraktische Verwirklichung des Horazischen "Multa renascentur quae jam cecidere" betrachtete. Doch war es nie meine Art, solche Neugeborene, auch wenn sie mir nicht lebensfähig erschienen, in der Geburt zu ersticken; man sieht aber aus der Art, wie ich mich in der ungedruckten Abhandlung und in dem bereits 1847 veröffentlichten kürzeren Artikel ausdrückte, dass ich der als neu betrachteten Methode kein glänzendes Horoskop stellte.

Was die in der hier folgenden Arbeit angeführten Namen Pariser Aerzte betrifft, so ist Malgaigne seitdem leider auch gestorben. Pépin hat gegen das Ende des Jahres 1848 Paris verlassen, um, wie man mir sagte, nach Amerika auszuwandern; ich konnte also diesen höchst unterrichteten und des Arabischen vollkommen kundigen Collegen über die dunklen Punkte meiner Arbeit nicht zu Rathe ziehen. Die Literatur dieses Gegenstandes hatte ich sehr vollständig vor Abfassung meiner Abhandlung durchforscht; daher ist auch seitdem nichts Neues über denselben zu meiner Kunde gekommen, wesswegen ich auch seine Form ganz unverändert lasse. Augenscheinlich wird es aber für alle Unbefangene sein, dass meine Abhandlung, zur Zeit ilırer Abfassung, nicht im Geringsten gegen Laugier gerichtet war, welchem die 
so alte Existenz seiner vermeintlich neuen Operationsmethode, zur 7eit wo er sie wiedererfand, vollkommen unbekannt geblieben war.

Paris, 18. October 1868.

$$
\text { Dr. Sichel. }
$$

\section{Historische Notiz uber die Operation des grauen Staares durch die Methode des Aussaugens oder der Aspiration.}

Von

Dr. Sichel in Paris.

In Malgaigne's ,Revue médico-chirurgicale”, 1847*), S. 18-26, hat Dr. Laugier, Wundarzt des Hospitals Beaujon, eine neue, von ihm erfundene Staaroperationsmethode beschrieben, welche er die Methode ,durch Aspiration" nennt. Sie besteht darin, dass eine hohle, auf eine den Stiel vertretende kleine Pumpe oder Spritze geschraubte Staarnadel durch die Sclerotica und die hintere Linsenkapsel in den unteren, äusseren und hinteren Theil der Staarlinse eingestossen wird, so dass die vordere Oeffnung des hohlen Ganges der Nadel sich im Innern des Staares befindet, und die Spitze des Instrumentes gleichfalls in der Höhle der Linsenkapsel ruht, ohne deren vordere Wand zu durchbohren. Die Nadel wird unbeweglich gehalten, und man macht einen luft-

*) Oder 1848; die betreffenden Bände meiner Bibliothek sind in Augenblicke verlegt. . 
leeren Raum, indem man sanft den Stempel der Spritze $i^{n}$ Bewegung setzt. Die weichen oder flüssigen Theile der Linse werden, nach La ugier's Angabe, durch die Nadel aspirirt und aus dem Auge gezogen, ohne dass man die vordere Linsenkapsel berührt. Genauer in den practischen und technischen Theil der Beschreibung des Erfinders einzugehen, ist für den $\mathrm{Z}$ weck unserer rein historischen Untersuchungen unnütz.

Nachdem Laugier diese Methode durch eigenes Nachdenken erfunden, forschte er nach, ob nichts Aehnliches von Andern vor ihm versucht oder ausgeführt worden sei, und fand eine Stelle in Albucasis' "Chirurgie", welche auf diese Methode Bezug hat. "Hier folgt", sagt Laugier (S. 24), "die Stelle des Albucasis, sowie sie Dr. Pépin in einer neuen Uebersetzung, deren Druck begonnen ist, nach dem besten Manuscript der königl. Bibliothek übertragen hat."

"Man hat mir gesagt," sagt Albucasis (Abulcasem), „dass ein in der Augenheilkunde unterrichteter Mann (un homme instruit dans l'oculistique), e in Bewohner Irak's, erzählte, er wende (qu'il employait) eine hoble Staarnadel an, vermittelst welcher er die Aussaugung des Wassers (aquam, so nannten die Araber den grauen Staar) bewerkstelligte. Ich habe niemals Jemand in unseren Gegenden (Albucasis wohnte in Spanien) ein solches Verfahren anwenden sehen, und ich habe es in den Schriften der Alten nicht angeführt gefunden. Es ist möglich, dass dies eiue neue Sache ist." -

"Dicselbe Stelle des Albucasis," fährt Dr. Lau gier fort, "ist in Haller's "Chirurgischer Bibliothek" folgender Massen übersetzt: Esse, qui ex Alayrach (forte Alkahirat, fœcundacaecorum nutrice) veniant, qui corneam tunicam perforent, atque 
aquam (lentem crystallinam) exsugant, quam administrationem negat, se vidisse aut legisse."

„Hier ist es also nicht mehr ein einziger Chirurg, sondern mehrere, welche, nach Durchbohrung der Hornhaut, den Staar ausgesaugt hätten. Es ist nicht die Rede davon, dass dies vermittelst einer hohlen Nadel geschehe, und Albucasis giebt ausdrücklich an, dass er nichts gesehen oder auch nur gelesen habe, was die Wirklichkeit eines ähnlichen Verfahrens beweise. Aber man kann vielleicht die Uebersetzung des Dr. Pépin, welche viel ausführlicher und bestimmter ist, als genauer ansehen."

$\mathrm{Zu}$ diesem historischen Theile von Dr. Laugier's Arbeit fügt der Redacteur der Zeitschrift, der geistreiche und um die Geschichte der Wundarzneikunst hochverdiente Dr. Malgaigne, noch folgende Details:

"Die Operationsweise durch Aussaugen scheint viel älteren Ursprungs $\mathrm{zu}$ sein. Man liest in der That bei Rhazes (Contin., lib. II, Tract. VI, cap. 2): Antyles dixit: et aliqui aperuerunt sub pupilla, et extraxerunt cataractam; et potest esse cum cataracta est. subtilis; et cum est grossa non poterit extradi, quoniam humor egrederetur cum ea. Et aliqui loco instrumenti posuerant concilum vitreum; et sugendo eam suxerunt albugineum cum ea."

„Nach Guy de Chauliac soll Avicenna dasselbe Verfahren erwähnt haben: Einige der alten Griechen, wie Albucasis und Avicenna angeben, machten eine Oeffnung unter der Hornhaut, und zogen, vermittelst einergehöhlten (cannelée) $\mathrm{Nadel,den}$ Staar durch Sangen (en suçant) heraus."

"Die lateinische Uebersetzung Avicenna's spricht nicht vom Saugen; es ist übrigens wahrscheinlich, dass wohl Guido ein Wenig aus dem Kopfe citirt hat; denn 
weder Avicenna noch Albucasis sprechen von den alten Griechen."

„Die Uebersetzung der Stelle des letzteren durch Dr. Pépin stimmt, nicht ganz mit den lateinischen Uebersetzungen überein. Hier ist zuerst die Gerard's von Cremona, welcher Haller gefolgt ist: Etiam quidem pervenit ad nos de quodam ex illis qui sunt de Alayrach quidixit quod factum fuit in Alayrach Magdaan perforatum quo sugitur aqua. Verum ego non vidi aliquem in terra nostra qui fecerit illud; neque legi illud in aliquoex libris antiquorum, et est possibile ut sit illud novum."

„Gleichlautend ist auch die lateinische Uebersetzung, Venet., 1500, in fol."

„Haller's Alnyrach ist ein Druckfehler; Alayrach bedeutet ganz einfach Irak oder Persien. Channing's Uebersetzung stimmt in diesem Punkte mit der Pépin's überein: Ex Iracensibus quis ad me venit quondam dixitque quod in Irak conficitur Makdach perforatum, quo exsugitur aqua. In regione nostra nunquam ejusmodi factum vidi, neque in aliquo antiquorum librovidi scriptum. Novum fortasse est inventum."

„Es kann Niemand entgehen, dass diese beiden vollkommen übereinstimmenden Versionen nicht von dem geschickten Augenarzte sprechen, den Pépin in der seinigen erwähnt."

Laugier's Erfindung und seine, sowie Malgaigne's im Vorstehenden angeführten historischen Bemerkungen, haben mich zu genaueren Untersuchungen über diesen interessanten Gegenstand, besonders nach den arabischen Quellen, veranlasst; welche für die Geschichte der Augenheilkunde vielleicht nicht uninteressant sein dürften.

Die Stelle des Abulkasem steht gegen das Ende 
des 23. Kapitels des 2. Buches seiner Chirurgie, ed. Channing, T. II, p. 172, lin. 2:

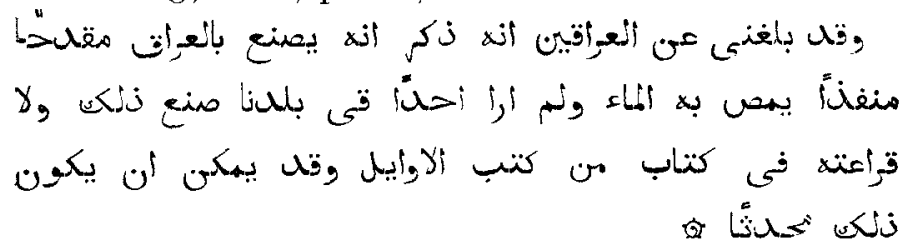

Zwischen den zwei ersten Worten den zwei folgenden ع fehlt offenbar das Wort ver, welches auch die vortreffliche Handschrift der hiesigen Bibliothek nicht hat; denn in diesem Manuscripte, welches Dr. Pépin aus meinen Händen erhielt, als er sich mit Albucasem zu beschäftigen anfing, habe ich alle auf Augenheilkunde bezüglichen Kapitel genau verglichen, und finde über die betreffende Stelle in meinen Noten keine verschiedene Lesart angemerkt. Ohne dies Wort (einer, ein gewisser) ist der Satz nicht correct. Auch findet sich in einer sehr guten hebräischenUebersetzung von Albuk a sem's Chirurgie, welche unter den Manuscripten der hiesigen kaiserlichen Bibliothek vorhanden ist, das streng gleichlautende Wort מקומת. Die Stelle lautet auf Deutsch folgender Maassen*): „Es ist mir zu Ohren gekommen, dass ein Bewohner Iraks gesagt habe, man verfertige in Irak eine hohle Staarnadel, mit welcher mandas Wasser aussauge. Ich habe Niemand in unseren Gegenden gesehen, der auf

*) Die ersten Worte können auf mehrere Weise übersetzt werden; ich erinnere mich, dass meine Uebersetzung von dem gelehrten, vor Kurzem verstorbenen Orientalisten M u n k gelesen und gebilligt wurde. Folgende, ungefähr gleichlautende, aber von der Pëpin's wesentlich verschiedene Version giebt mein Freund Dr. L. Leclerc (L a C h ir urgie d'A buleasis, Paris, 1861, in $8^{\circ}$, p. 93): „J'ai rencontré un Persan, qui m'a affirmé que dans son pays on fabriquait un mikdah perforé, au moyen duquel on aspirait la cataracte. Je n'ai rien vu faire de tel chez nous, et je n'ai rien lu de pareil dans les écrits des Anciens. Il est possible que ce soit une invention récente." 
diese Weise operirt habe, noch habe ich es in irgend einem der Bücher der Alten gelesen. Es ist möglich, dass dieses etwas Neues ist."

Man sieht aus der Vergleichung des Originals mit Dr. Pépin's Uebersetzung, dass er in der ersten Linie des Textes die Genauigkeit der Eleganz des Styls geopfert, indem er die Worte "ein in der Augenheilkunde erfahrener Mann" willkührlich eingeschoben; dass er ferner den Satz „dass man in Irak eine hohle Nadel verfertigt (انغ بُمنَعُ بالعرأث), im hebräischen MS.

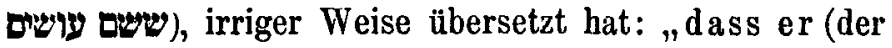
Augenarzt) eine hohleNadel an wen dete", als stände يصنع im Aktivum und ohne das Wort بالعرأق. (Der Akkusativ ist in dieser Construction ein häufiger Fehler der Abschreiber.) Ein neuer Beweis, wie wichtig es ist, sich in Uebersetzungen immer so streng als möglich an den Text zu halten, besonders wenn es sich darum handelt, den Schleier zu lüften, welcher die frühesten schwankenden Schritte unserer Kunst in Dunkel hüllt. Der Widerspruch zwischen Pépin's Version und der seiner Vorgänger, so wie das Auffallende der von ihm in den Text gesetztenErwähnung eines geschickten A u gnarztes, ist Malgaigne's Scharfblick nicht entgangen. Was Malgaigne's Bemerkung über Guido's Citation betrifft, so hat der Chirurg von Chauliac in Bezug auf Albukasem allerdings vollkommen Recht zu sagen, er spräche von den alten Griechen. Denn das Wort: ,die Alten" (الاروايل) bezieht sich bei den Arabern bekanntlich, mit wenigen Ausnahmen, auf die Griechen.

Beim Durchblättern einer sehr vollständigen arabischen Handschrift (Pariser kaiserl. Bibliothek Nr. 1100), des Buchs über die Augenkrankheiten von Isa-ben-Ali*),

* Von dem Werke dieses Augenarztes hat Dr. Hille jun. in Dresden eine Auggabe unter der Feder, von der bereits ein Prodromu 
der im neunten Jahrhundert Augenarat zu Bagdad war, fand ich vor wenigen Tagen, im Rande des Kapitels über den grauen Staar, eine kurze, aber höchst wichtige Note über die Operationsmethode durch die Aussaugung, mit einer Zeichnung, von der ich nebenstehend eine getreue Copie gebe.

Sie stellt, wie man sieht, eine hohle Nadel vor, die an ihrem nnteren Fnde, d. h. an dem der Spitze entgegengesetzten Theile des Stieles, offen ist. Die Note selbst ist nur die Erklärung der Figur und lautet folgender Massen:

$$
\begin{aligned}
& \text {, }
\end{aligned}
$$

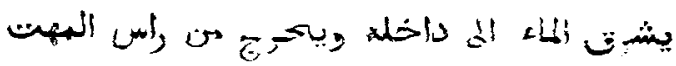

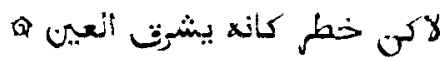

Dies ist die Gestalt der hohlen Staarnadel, welche man die Chorasanische nennt. Sie zieht das Wasser (den Staar) in sich (in ihre Höhlung), und entleert es durch den Kopf der Nadel (durch das Ende des Stieles). Doch ist (diese Operationsweise) mit Gefahr verbunden, denn sie könnte wohldas A u ge (durch Auslaufen der normalen Flüssigkeiten) entleeren.

erschienen. Ueber diesen sowohl, sls ïber die Ausgabe celbst, habe ich im Journal asiatique, 1842, und in den Annales d'Oculistique, t. 18, 1847, p. 230, berichtet. 
Die Uebersetzung dieser Erklärung ist nicht ohne Schwierigkeit. H (Mihatt) ist eine Staarnadel, scheint aber, nach Avicenua, T. I. p. 353, ed. Rom., mit هقد (Mikdahh) nicht ganz gleichbedeutend. Wie dies letzteWort

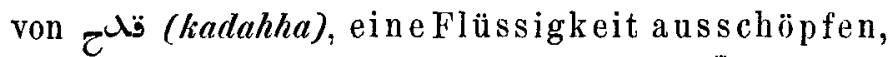
einen Brunnen reinigen, kommt das erste von هن (hatta), eine Flüssigkeit ausgiessen. Beide sind also von dem Grundbegriffe des Ausleerens einer Flüssigkeit hergeleitet, auf welchen wir weiter unten zurückkommen werden. Freitag, in dessen Wörterbuche leider viele medicinische Kunstausdrücke fehlen, übergeht unter derWurzel هـ هort gan Wor, welches doch offenbar keinen anderen Ursprung haben kann, obgleich $\mathrm{Ca}$ stell es irriger Weise unter مبت gebracht. Meninski hat unter diesem und dem folgenden Worte nichts, was die Frage weiter fördern könnte.

Unter den Bedeutungen von شتى tag keine, die einen ganz klaren und befriedigenden Sinn für obige Note gäbe. Aus dem Begriffe des Aufgehens der Sonne kann man den des Hervortretens, Hervorquellens ableiten; und so habe ich denn, dem Sinne der Stelle nach, die zweite Form dieses Zeitwortes in der Bedeutung von hervorquellen machen übersetzt. Die Nadel macht den Staar nach innen, d. h. in ihr Inneres, in ihre Höhlung, hervorquellen; die Operationsmethode ist gefährlich, da sie das Auge, d. h. seinen flüssigen Inhalt, hervorquellen machen kann. Vielleicht kann man eine ähnliche Erklärung aus einem anderen Grundbegriffe des Wortes "decerpsit fructum" ableiten: „Die Nadel bricht den Staar ab, wie eine Frucht vom Baume, zieht ihn hervor und heraus"; was mir aber, 
da es sich von einer Flüssigkeit handelt, noch weniger natürlich scheint als die erste Auslegung. Gleichsam alsob sie, ist wohl ein Schreibfehler für ${ }^{\prime}$, weil sie das Auge entleeren kann. Der Vorwurf der Mög. lichkeit des Auslaufens der Augenflüssigkeiten findet sich auch bei Rhazes (s. oben S.5).

Bei der Spitze der Nadel finden sich auf der Abbildung die Worte: "Dieser Theil wird in's Auge e ingeführt." Beim offenen Ende des Stieles liest man: ,Durch diesen Ort steigt das Wasser herab" (d. h. läuft aus).

Ohne allen /weifel ist die Zeichnung und die Beschreibung dieser Nadel nicht von Isa-ben-Ali; denn beide stehen im Rande des 73. Kapitels des zweiten Buches (Cap. 67 dex lateinischen Uebersetzung), in welchem das Allgemeine über den Staar theoretisch abgehandelt wird: und in folgenden Kapitel, wo von der Operation die Rede ist, wird die Methode des Saugens weder in der Pariser, noch in der Dresdener Handschrift, noch in der lateinischen Uebersetzung des Mittelalters (z. B. Venet. 1500 in fol.) auch nur mit einer Sylbe erwähnt. Dennoch ist diese Stelle von der grössten Wichtigkeit, besonders wenn man den Namen Chorasanische $\mathrm{Na}$. del, der ihr hier mit so grosser Bestimmtheit und gleichsam als etwas allgemein Bekanntes beigelegt wird, mit den oben (S.5) angeführten Worten des Continens und deuen Abulkasems vergleicht. Rhazes, bekanntlich aus Raï in Chorasan gebürtig, spricht auch von der Aussaugung als von etwas, wie es scheint, zu seiner persönlichen Kenntniss Gelangtem. Da Chorasan eine an Irak grenzende Provinz Persiens ist, so darf man wohl aus der Vergleichung der drei Stellen die Schlussfolgerung ziehen: die Methode der Aussaugung der Cataracte sei wahrscheinlich vor dem neunten Jahrhundert in Persien 
häufig ausgeübt worden und vielleicht sogar wăhrend einer gewissen Zeit ziemlich allgemein in diesem Lande verbreitet gewesen.

Was die bereits oben (S. 5) angeführte Stelle des Continens des Rha z es betrifft, so muss das unverständliche und wahrscheinlich corrupte Wort concilum dem Sinne nach eine Röhre, einen Tubulus bedeuten. Leider besitzt die hiesige Bibliothek unter ihren unzähligen arabischen $\mathrm{Ma}$ nuscripten kein Original des Elhhâvi; doch hat sie davon eine handschriftliche lateinische Uebersetzung (6912, infol.); in dieser (fol. 79, verso, zu Anfange der zweiten Spalte) wird das gleichfalls unverständliche couellum, welches ebensowenig als concilum in Ducange's Gloss. med. et infim. latinit. angeführt ist, durch die im Rande beigeschriebene Variante: „alii canulam" hinlänglich erklärt, und concilum, so wie couellum sind wahrscheinlich nur Schreibfehler für canulam. Die Worte: "loco instrumenti posuerunt" sind meines Erachtens nicht so $\mathrm{zu}$ verstehen, als hătten die diese Methode anwendenden Wundärzte anstatt des Staarinstrumentes ein, etwa spitziges, Röhrchen eingebracht, sondern vielmehr so, dass sie in den Ort des Instrumentes, womit die Hornhaut unter der Pupille eröffnet worden, das heisst in die Oeffnung der Cornea, die Röhre einführten. Denkt man sich die angeführten

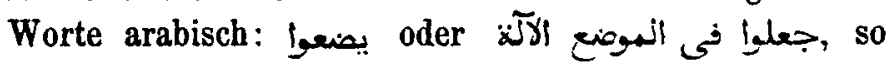
findet man in dem lateinischen Satze die vollkommen wörtliche Uebersetzung, und man weiss ja, dass die Uebersetzer des Mittelalters das buchstäbliche Wiedergeben der arabischen Originale bis zur Unverständlichkeit trieben.

Die Worte: Et aliqui, beweisen deutlich, Rhazes habe nicht daran gedacht, die Aussaugung dem Antyllus als Erfinder zuzuschreiben. Auch bei keinem anderen griechischen Schriftsteller ist von dieser Methode, meines Wissens, die Rede. Wenn also Billaudeau (Gazette 
des hôpitaux, 1847 , n. 9 , p. 38) ohne Weiteres sagt, die Aspiration des Stars stamme von den Griechen her, so muss ich dies, bis er seinen Gewährsmann anführt, für einen Irrthum halten. Dass Rhazes, der selbst die operative Augenheilkunde nicht practisch ausübte, seiner Landsleute nicht erwähnt und nichts Genaueres über diese persische Verfahrungsweise angiebt, kann nicht auffallen.

Die Griechen kannten die Aussaugung des Staares nicht; sie sprachen bloss von seiner Ausziehung. Antyll us (bei Rhazes, s. S. 5) beschreibt diese letztere. Auch Galen spielt auf die Extraction an in folgender Stelle, welche nicht auf die Suction bezogen werden kann (Meth. med., lib. XIV. c. 13, t. X, p. 987, ed. Kühn): „Einige versuchten auch, den Staar zu entleeren (xøvoũv), wie ich in den (nicht auf uns gekommenen) Büchern über Chirurgie sagen werde."

Die Aussaugung wurde also von den Einen (nach Abulkasem und der Note bei Isa-ben-Ali) auf die gewöhnliche Weise, $d . h$. durch die Sclerotica, von den Anderen durch die Hornhaut, verrichtet.

Ist hier nicht das bekannte: Multa renascentur quae jam cecidere, ganz an seinem Orte? Und wenn ein denkender und höchst erfahrener Wundarzt eine Operationsmethode erfindet, welche, ihm unbewusst, bereits vor zehn Jahrhunderten in einer bedeutenden Provinz ziemlich allgemein ausgeübt wurde, liefert dieser Umstand nicht allein schon einen hinreichenden und praktischen Beweis für die Wichtigkeit des Quellenstudiums der Geschichte unserer Wissenschaft? Es ist dies nicht das erste Mal, dass in den Alten, deren Werth ich weit entfernt bin zu überschätzen, und deren Werke wir ja keineswegs lesen, um uns zu ausübenden Aerzten zu bilden, etwas vermeintlich Neuerfundenes vollkommen entwickelt angetroffen wurde. Muss man also nur gleich mitleidig 
lächeln oder verächtlich die Achseln zucken, wenn ihre medicinischen oder chirurgischen Heilmethoden auch aut den ersten Anblick manchmal ein wenig absonderlich oder roh erscheiuen?

Sucht man sich nun zu erklären, auf welche Weise die Araber auf die Operationsmethode des grauen Staars; durch die Aussaugung verfallen seien, so muss man ja nicht glauben, es habe sie eine genaue Kenntniss der verschiedenen Consistenz der Linseustaare dahin geführt. Allerdings war ihnen bekannt, dass ein Staar von grösserem Umfange und von bedeutenderer Dichtigkeit sei, als der andere; doch wussten sie hierüber Nichts, als was sie von den Griechen gelernt hatten. Man muss es vielmehr ihrer Nomenclatur der Cataracte und einem derselben zu Grunde liegenden anatomischen und pathologischen Irrthume zuschreiben, dass sie Versuche machten, den Staar durch Saugen aus dem Auge zu schaffen. Es ist bekannt, dass die Griechen, welchen die Araber in der Anatomie und Yathologie fast ausschliesslich folgten, den Linsenstaar Glaukom ( $\left.\gamma^{\lambda} \alpha v^{\prime} \varkappa \mu \alpha\right)$ nannten und als unheilbare Blindheit betrachteten, weil die Linse für sie das unmittelbare Werkzeug des Gesichtes war; dass sie den operirbaren grauen staar hingegen als einen Erguss oder eine Ausschwitzung einer wenigstens zuerst flüssigen Materie (vंó $\% v \mu a$, suffusio) ansahen. Die Araber nahmen diesen ungegründeten und sehr spät erst durch die pathologische Anatomie widerlegten Begriff auf, und nannten diesen, als das Product eines Ergusses betrachteten grauen Staar bald das Niedersteigen oder Niedersinken des Wassers im Auge, oder die Ansammlung von Wasser im Auge (أنساء النبازل نى العبن), bald das im Auge niedergesenkte, herabgestiegene oder angesammelteWasser (النترول الماء فى العبن), oder abgekürzt, das Wasser (ألساء). Die Operation des Nieder- 
legens der Cataracte wurde daher, wie schon gesagt, das Ausschöpfen (ov) oder Ausgiessen (هن) des Wassers genannt. Später wurde dises Niederfallen des Wassers, als ein wirklicher Wasserfall, mit catarrhacta, cataracta (※orregurns) übersetzt, welches Wort bekanntlich bei den Griechen und Römern in der Bedeutung des Staars nicht vorkommt, und der Ursprung des allgemein angenommenen Namens cataracta ward.

Dass nun unter so Vielen, welche dieses vorgebliche Wasser mit chirurgischen Werkzeugen zu heilen versuchten, sich auch Einige fanden, welche auf den Gedanken geriethen, es möchte wohl besser sein, diese trübe Flüssigkeit durch Aussaugen wirklich ganz aus dem Auge zu schaffen, hat nichts Allzuüberraschendes; mir wenigstens scheint es, dass dies in der damaligen Zeit natürlicher war als heutzutage, wo wir doch an Staaroperationsmethoden, deren Erfolg sicher genug ist, keinen Mangel leiden. Diese Aussaugungsversuche, bei welchen, nach den Einen, eine Nadel durch die Sclerotica eingeführt, nach Anderen, wie uns Rhazes überliefert, durch einen Einstich an dem unteren Theile der Hornhaut eine feine Röhre eingebracht wurde, können als eine Annäherung zur Ausziehung des Staares, wenigstens zur theilweisen, betrachtet werden, obgleich schon Antyllus viel früher der Extraction Erwähnung thut.

Galeatius de sancta Sophia (opus medicinae practicae.... in nonum tractatum libri Rhasis ad Regem Almansorem ....., Haganoae 1533, fol.) hat später die Operationsweise des Staars durch die Aussaugung folgendermassen ausführlich beschrieben (fol. 2\%, verso): Vel aliter fiat hic modus magistralis (der Staaroperation), quem ego Galeatius de sancta Sophia jam duduin imaginatus fui. Primo ergo accipiatur una acus aurea per totum subtiliter concavata prope cuspidem, et dicta acus sit perforata usque ad concavitatem foraminis 
parvi. Quo facto perfora oculum ut supra dictum est, et cum apposuisti acum intra illam aquam, tunc volve dictam acum bis vel ter, et post ea extrahe flatum a superficie acus superiori fortiter ad hoc ut' dicta aqua ingrediatur concavitatem acus. Et si tota aqua ingredi non posset, acum bis vel ter trahendo, tunc bene ipsam preme inferius ut nihil remaneat. Nam sic extrahendo dictam aquam extra totum oculum est cura caeteris perfectior, qua amplius dicta materia reverti non potest ad pupillam. Dico tamen quod haec cura fieri debet per Medicum valde peritum in hac arte. Et quamvis hanc curam hoc modo fieri non vidi, ipsam tamen posui, quia mihi possibile videtur esse. Facta igitur evacuatione illius materiae facientis cataractam extrahendo ipsam ad extra, sive premendo intra corneam et uveam, tunc accipiatur albumen ovi cum vitello etc. (Hier folgt die gewöhnliche Nachbehandlung.)

Wie man sieht, giebt sich Galeatus als den Erfinder dieser Methode an; doch scheint es mir sehr unwahrscheinlich, dass er vor Abfassung seines Commentars über das neunte Buch von Rhazes Almansor nicht vorher, zum besseren Verständniss, wenigstens die betreffenden Kapitel von dessen Continens gelesen haben sollte. Auch gesteht er selbst, diese Operation nicht verrichtet zu haben, was die Aufrichtigkeit seiner Angabe noch unwahrscheinlicher macht; denn wer das Bedürfniss einer neuen Operation fühlt, versucht sie auch gewöhnlich gern zuerst.

Galeatius war übrigens nicht der Einzige, der sich die Erfindung dieser persischen Staaroperation zuschrieb; er hat in Rochus Mattioli einen Concurrenten, dessen Verfahren Borri vervollkommnet zu haben vorgiebt. Der mailändische Edelmann, Goldmacher und gewiss auch Charlatan, Franz Joseph Borri, welcher 
gegen die Mitte des 17. Jahrhunderts durch seine vorgebliche Frfindung, den Wiederersatz der Brechungsflüssigkeiten des Auges uach ihrem Ausfliessen durch ärztliche Heilmittel zu bewirken, grosses Aufsehen erregte, und zuletzt in den Gefängnissen der römischen Inquisition sein Leben endete; Borri, der unter dem Namen Burhus bekannter war, sagt hierüber Folgendes in seinem Buche: Fipistolae ad Bartholinum artificio oculorum humores restituendi, Hafn. $1669,4^{0}$, p. 51, sqq. Rarum instrumentum ad deponendas oculorum Cataractas inventum a Nobili Domino Roceho Mattioli, Chirurgo Italo olim a servitiis (p. 52). A rchiducis Ferdinandi Caroli Austriaci... Artifex iste excogitaverat arundineam acum desinentem in vacum mucronem, qua penetrare oculos et per eam oris suctu extrahere posset cataractas; sed cum exilis orificii angustia et crassities membranae sugendae solertiam ingeniosi artificis remorarentur in perficienda operatione, ipsi Auctor fui includendi suo tubulo aeneo acuminato in modum tenuis penicilli subtilissima Auri textilis filamenta, quae in girum acta $\mathfrak{a}$ contorquentibus digitis, dum (53) acus tenebat medium orbitae, educerentur quasi ex aenea vagina et humorum instar membranam vel interciperent vel omnino in frustilla discerperent, atque iterum post hoc officium retrahi possent ad angustias sui aenei integumenti. Votum eventus felicissimus beavit, quia cataractae per hoc instrumentum depositae non ultra assurrexerunt ad eclipsandam lucem radiorum, cum alioquin eae quae a vulgari acu demittuntur paulatim a motu oculorum, ut nondum divulsae a suis radicibus, eriguntur. Die sehr undeutlichen Worte: et humorum instar etc. sind, Archiv fir Ophthalmologie, XIV. 3. 
glaube ich, so zu verstehen, dass die spitzigen und schneidenden Goldfäden (d. h. feine Golddräthe), welche den Pinsel bilden, beim Auseinanderfahren die Linsenkapsel entweder spannen, wie es die in ihr enthaltene Flüssigkeit (ein flüssiger Staar) thun würde, oder dieselbe zerstückeln. Die Hinzufügung des Golddrathpinsels zur Saugnadel hat Chelius (traité d'ophthalmologie, t. II, p. 274, n. 5) irriger Weise Mattiolus zugeschrieben. Borri giebt (p. 65, fig. I u. II) die Abbildung des von ihm erfundenen Metallpinsels und des zugespitzten Röhrchens.

Die Schriftsteller des 15., 16. und 17. Jahrhunderts, welche auf die Erfindung der Staaraussaugung Anspruch machen, verdienen auch desswegen schon keinen Glauben, weil ihre Zeitgenossen diese Methode, so wie die Ausziehung, häufig, als von den Griechen und den Arabern verrichtet, erwähnen. Man vergleiche z. B. folgende zwei Stellen noch im vergangenen Jahrhundert vielgelesener Schriftsteller.

Arculanus, des Rhazes Commentator in der Mitte des 15. Jahrhunderts, spricht sich folgender Massen aus (Joh. Arculani, Practica, Venet. $1557,{ }^{*}$ ) in fol., c. 30 ad fin., p. $58 \mathrm{E}$ ): Aliqui tamen ex Graecis antiquis, ut recitat Albucasis et Avicenna, faciebant foramen subtus, scilicet corneam, quod forte melius erat facere in conjunctiva cum acu canulata, ut sugendo extrahant cataractam, quae operatio est inconveniens, nisi aliquibus observatis conditionibus. Prima, ut aqua ipsa sit citra uveam et non ultro. Secunda quod illa aqua sit fluxibilis non confirmata, et quod operatio fiat paulatim, ne simul albugineus extrahatur. Et haec est propria cura saniei retro

*) Oder 1596. Die Jahreszahl meines Manuseripts ist bei einer Feuersbrunst, sowie anch das Wort er at und einige andere Worte des Randes, verbrannt. 
corneam. Die letzten Worte bedeuten, die Operation werde so verrichtet, wie die des Hypopyon, d. b. durch Eröffnen der Hornhaut mit dem Instrumente.

Andrea della Croce (Chirurgia universale e perfetta, Venezia, 1573*), in fol., p. 220) erwähnt gleichfalls die bereits angeführte Stelle Abulkasem's, aber auf eine solche Weise, dass man seine eigenen Worte nicht streng von denen des arabischen Wundarztes unterscheiden kann. Zugleich schiebt er diesem letzteren eine specielle Indication für die Staaraussaugung unter, an welche jener nicht gedacht hat. Se vi sara humore di sangue o escreszentia di carne [diese Worte finden sich nicht bei Abulkasem], dice Albucasi che alcuni ritrovarono uno stilo forato (detto da lui Magdaam), col quale si succhia commodamente l'acqua; della qual cosa pero nonne havendo gl'Antichi fatto mentione alcuna giammai, non so io quello che dir mi debbo; riservandomi certo a farne un giorno la pruova, e maggiormente che alcuna volta mi sono anco da me stesso cotesta operatione imaginata. Also auch della Croce hatte die Aussaugung erfunden, ohne seine Vorgänger gekannt $\mathrm{zu}$ haben. Doch ist er ehrlich und aufrichtig genug, diese letzteren $z u$ nennen, und sich die Operation nicht zuzuschreiben, was er eben so gut, als Galeazzo, Rondelet u. s. w. hätte thun können, welchen gewiss die arabischen Urerfinder der Methode nicht vollkommen unbekannt sein konnten.

$\mathrm{Zu}$ den vorgeblichen Erfindern der Staaraspiration gehört auch Rondelet, Professor der Medicin zu Montpellier im 16. Jahrhundert. Si cataracta, sagt er (Gulielmi Rondeletii Method. curandor. morbor.... Paris, 1575, in $8^{\circ}$, p. 135 , verso, ad fin.), sit in prin-

*) Siehe die vorhergehende Note. 
cipio adhuc, et tantum sit aqua, vel aqueus quidam vapor appareat, potest resiccantibus et digerentibus medicamentis curari, qualia sunt. ....Posset etiam curari manu, si componatur tale intrumentum. Fiat acus cava, instar syringae, et, ut facilius disecet corneam, et subingrediatur, paretur, ut calamus scriptorius, mittatur intra oculum, exactissime jungatur vulneri, nec aër attrahatur, et sugendo acum attrahat per eam ex oculo aquam. Dies ist die von Rhazes angegebene Operation durch die Hornhaut, nur dass die Cornea nicht erst mit einem andern Instrumente geöffnet, sondern gleich mit der bohlen röhrenförmigen Staarnadel von Chorasan, oder mit einem, wie eine Schreibfeder zugespitzten und an den Seiten der Spitze schneidenden Röhrchen, angestochen wird. Nähme man das Wort syringa, mit seiner aus dem Zusammenhange gerissenen Umgebung, in dem Sinne einer Spritze, welchen es heut zu Tage in der Wundarznei hat, und mit welcher Bedeutung es auch in die französische Sprache übergegangen ist (seringue), so könnte man darin die erste Anwendung der, an Laugier's Instrument angebrachten Spritze oder Pumpe zur Staaroperation finden wollen. Dies wäre aber offenbar ein Irrthum; denn syringa ist hier augenscheinlich gleichbedeutend mit dem griechischen ov́ory, fistula, eine Röhre. So haben es $z$. B. die beiden sogleich anzuführenden Schriftsteller verstanden, wie ihre Vergleichung dieses Röhrchens mit dem Catheter beweist. Rondelet hat zweifelsohne von Mattioli seinen zugespitzten Tubulus entlehnt.

Claudini, der Ende des 16. und Anfangs des 17. Jahrhunderts zu Bologna lebte, billigt Rondelet's Operation nicht. Ueber diese, so wie über die Staarausziehung, spricht er sich folgender Massen aus (J. C. Clau- 
dini Empiric. rational. Bonon, 16533, p. 467, ad fin.): Sed praeter has materias chiruruicas (Schröp. kỏpfe u. s. w.), in cataracta absoluta necessaria est punctio, qua mediante, si materia tenuis ac fluxilis existat, evacuatur sensibiliter; si vero crassa, acu deponitur. Non ignoro, Gal. 14. Meth. et Rhas. 2 Continentis affirmare fuisse quosdam qui etiam crassam materiamtentarunt punctione aon transponere, sed evacuare ex extrahere. lerum quia hic est maximum periculum, ne simul evacuetur humoralbugineus, cum necessario in tali casu foramen latius excitari debeat, iccirco parvi facio eam operationem. Nec [der Sinn erfordert: Nec non] et illam Rondeletii, qui parva syringa oculis accommodata putat sensibilen patrari posse evacuationem, al similitudinem ejus, quae in virgam immittitur, et per eam impositam loco, ubi alias admonetur pro transpositione, ore attrahenda materia; iisdem enim facultatibus est obnoxia. [Rondelet's Methode sei denselben Zufällen ausgesetzt als die Extraction und daher, gleich ihr, zu verwerfen.] Porrofit punctio, ut in materia fuxili evacuanda, eodem modo, et loco, quo in hypopyo fuit monitum. Claudini hat Rondelet missverstanden; obgleich der letzte Satz, durch die Vergleichung mit der Operation des Eiterauges, Rondelet's an sich schon klare Angabe der Punktion durch die Hornhaut noch klarer in's Licht setzt, so spricht Claudini doch noch von dem Einstiche an dern bei der Niederlegung (transpositio) gewöhnlichen Orte.

Hercules de Saxonia, Claudini's Zeitgenosse, führt gleichfalls Rondelet's Operationsweise an (Hercules Saxonia, Pantheum medicinae selectum, Francofurt, 1603 , in fol., 1. I, c. 9 , p. 128, ad fin). In suffu- 
sione, quae fit a materia adhuc in membranam non conversa, atque ea quidem crassa a dmodum et lenta; ... materia omnino est attenuanda... (p. 129.) Ubi attenuata est materia, digerenda est ex sententia R ondeletii, et etiam evacuanda: scribit ille, syringem conficiendam esse parvam oculo accommodatam in similitudinem illius, quae in virgam immittitur, vultque illam imponi oculo eo modo quo acus in depositione suffusionis, et orevult attrahi aquam. Utrum ille feliciter sit expertus, ignoro, ego nunquam probavi: forte excogitavit potius quam expertus est. Claudini scheint Rondelet's Worte nicht selbst gelesen, sondern bloss Saxonia abgeschrieben zu haben. Dass einer der zwei italienischen Professoren wenigstens den andern copirt habe, geht daraus hervor, dass der Irrthum über den Einstich in die Sclerotica und die von Rondelet nicht gemachte Vergleichung der Röhre mit dem Catheter bei beiden mit denselben Worten vorkommt. Dass Saxonia statt des von dem französischen Arzte gebrauchten Wortes syringam das richtigere und unzweideutige syringem setzt, würde, wenn dies nöthig wäre, noch strenger beweisen, dass von einer Spritze nicht im Geringsten die Rede ist. Saxonia's Bemerkung: forte excogitavit potius etc. ist ganz richtig. Hätte Rondelet seine Methode an Lebenden oder auch nur an der Leiche versucht, so würde er wohl statt: Posset etiam curari maru, gesagt haben: Potest.

Lamzweerde (Append. ad Sculteti Armament. chirurg., Amst., 1741, in $8^{\circ}$, T. II, p. 413-433) hat Borri's und Bartholin's Briefwechsel abgedruckt, und zu des ersten Abbildungen und Beschreibung Zusätze geliefert (tab. XIV. u. pag. 433 sq.), welche beweisen, dass er auf diese Operationsweise grosse Hoffnungen setzte. 
Nachdem das Röhrchen durch die Hornhaut eingeführt und der Staar ausgesaugt worden, solle man, sagt Lam zweerde, in das Röhrchen den goldenen Pinsel einschieben, durch Drehen des Kopfes oder Stieles desselben die gleichsam lancettförmigen Goldblättchen zum Auseinanderweichen bringen, so den Staar sanft einschneiden und abschaben (leniter incidant et abradant), und dann von Neuem und bequemer aussaugen. Nöthigenfalls wiederhole man die Operation, bis vom Staare nichts mehr übrig bleibe. Dieses Verfahren erfordere Geduld von Seiten des Kranken, sei aber sicherer, da es das Wiederaufsteigen der Cataracte verhüte; die Einschneidung (sectio) der Hornhaut sei übrigens ohne Gefahr. Er empfehle allen Chirurgen diese Methode, obgleich sie unerhört (inaudita), d. h. nagelneu sei. Es bleibt das Neue stets ein relativer Begriff, und vieles schon Vorhandene wird erfunden, wenu man nicht der Mühe werth hält, im Längstbestehenden, Veralteten und Vergessenen nachzuforschen.

ls kann keinem Zweifel unterworfen sein, dass Rochus Mattioli (oder Mattiolo), ein sonst unbekannter Wundarzt, welchen nan nicht mit dem, um ungefähr ein Jahrhundert früheren berühmten Arzte und Botaniker Peter Andreas Matthioli verwechseln darf, seine vorgebliche Erfindung von den Arabern und von Rondelet entlehnt habc.

Der Vollständigkeit wegen sei hier noch erwähnt, dass ein Ungenannter (l'Union médicale, 1\&47, no. 15, p. 60) für Prof. Pecchioli in Siena die Erfindung der Staarnadel mit der Pumpe in Anspruch nimmt; dass aber Dr. Laugier (ibid. n. 17, p. 66) mit Recht bemerkt, Charrière's Instrumentenverzeichniss vom Jahre 1835 gebe zwar bei den Augeninstrumenten unter Nr. 6 eine ,aiguille à pompe de M. Pechioli" an; eine Beschreibung 
derselben, so wie eine Angabe ihrer Bestimmung und Anwendungsweise, finde sich aber nirgends vor. ${ }^{*}$ )

Was nun die Aspirationsmethode betrifft, wie sie der geschickte Chirurg des Hospitals Beaujon ausübt, so muss ich gestehen, dass ich sehr bezweifle, sie werde, neben der Ausziehung, der Niederlegung und der Zerstückelung, ein dauerhaftes Bürgerrecht in der Augenheilkunde behaupten. Auf jeden Fall ist es offenbar, dass weder die harten noch die weichen oder selbst halbweichen Linsenstaare durch dieses Verfahren mit glücklichem Frfolge operirt werden können, und dass sie bloss bei flüssigen Cataracten ihre Anwendung finden kann. Aber wird selbst in diesen die vordere Linsenkapsel, nach Ausleerung der in ihr enthaltenen undurchsichtigen Flüssigkeit, nicht immer einen Nachstaar bilden, und eine

*) Folgende Notiz, welche beweist, dass Pecchioli's Instrument allerdings zur Staaraussaugung dienen kann, und auch vielleicht dazu bestimmt war, ist mir erst später zu Augen gekommen. Da sie in einem Fenilieton rersteckt lag, wo sie mir, trotz meiner Gewohabeit, alles Augenheilkundige in meinen Exeerpten zu notiren, gänzlich ent gangen war, so ist es nicht zu verwundern, dass auch Dr. Laugier sie nicht gekannt hat.

Gazette médicale de Paris, 1838, n. 1, p. 3: Fragments d'un voyage médical en Italie, par Petrequin. M. Pecchioli, de Sienne, a inventé pour l'opération de la cataracte une aiguille, qu'il propose d'employer dans quelques circonstances; c'est un instrument semblable à celui de Scarpa, avec cette différence que la tige est creusée d'un petit canal, et que le manche renferme un piston destiné à faire le jeu d'une pompe aspirante. Ce vide qu'on forme dans le conduit central et le manche, aspire les liquides en contact avec l'orifice de la tige placé vers la lance.

On pourrait employer cette aiguille dans les cataractes fluides, qui troublent, en s'épanchant, la vue de ce qui se passe dans l'œil qu'on opère; elle agit, du reste, et peut servir comme l'aiguille ordinaire à abaissement.

Aus den vorhergebenden Zeilen scheint mir nicht herrorzugehen, dass $P$ ecchioli sein Instrument am Lebenden angewand, noch dass er bei seiner Erfindung ausschliesslich und bestimmt an die Staaraussaugung gedacht habe. 
neue Operation erheischen? Ich meinerseits befürchte es sehr, da ich nie nach einer Staaroperation die zurückgebliebene vordere Wand der Kapsel ihre Durchsichtigkeit längere Zeit behalten sah. Nichts destoweniger habe ich Laugier's Abhandlung mit gro:sem Interesse gelesen, und will das Verdienst seiner Methode auf keine Weise schmälern, noch vielweniger die Fortsetzung seiner Versuche und genauen Beobachtungen am Lebenden missbilligen. Vielleicht führt diese, trotz meiner oben gemachten Einwürfe, zu einigen speciellen Indicationen in bestimmten Fällen, wo die übrigen bis heute bekannten Verfahrungsweisen keinen oder wenigstens keinen so guten Erfolg haben.

Während der Correctur dieses Bogens schrieb ich an Laugier, um von ihm selbst zu erfahren, welchen Erfolg er seit zwanzig Jahren von seiner Operationsmethode beobachtet, und ob er dieselbe beibehalten und vervollkommnet oder aufgegeben; er war aber gerade verreist. Vielleicht giebt er mir später eine, zu Ende des gegenwärtigen Bandes einzurückende Note über das Resultat seiner Erfahrung, welche gewiss diese rein hi- . storischen Untersuchungen auf eine praktische Weise vervollständigen wird. 